\title{
A psicologia teórica e filosófica como uma área de pesquisa acadêmica
}

\author{
Philosophical and theoretical psychology as a field of academic research
}

\author{
Flávio Fernandes Fontes ${ }^{\mathrm{I}}$ \\ Jorge Tarcísio da Rocha Falcão ${ }^{\mathrm{I}}$
}

\section{Resumo}

Pesquisas filosóficas e conceituais vêm sendo reiteradamente apontadas como necessárias na Psicologia, dada a caracterização da disciplina como excessivamente centrada no método e na coleta de dados empíricos. Em resposta a isso, ocorreu ao longo das últimas décadas o surgimento de uma nova área de pesquisa, a Psicologia Teórica e Filosófica. Com o objetivo de fornecer uma introduçáo a este campo, são destacados alguns dos principais periódicos, programas de pós-graduação e instituiçóes que produzem conhecimento nesta vertente. Procurou-se delimitar o escopo e o objetivo da Psicologia Teórica e Filosófica. Foram revisados diferentes autores que procuraram estabelecer quais as suas principais atividades, para isso foram elencados alguns exemplos de pesquisas importantes, e concluiu-se a relevância do fomento deste tipo de pesquisa para a Psicologia como um todo.

Palavras-chave: psicologia teórica; psicologia filosófica; análise conceitual.

\begin{abstract}
The need for philosophical and conceptual psychology research has been repeatedly pointed out due to the characterization of the discipline as excessively focused on method and empirical data collection. In the last few decades, a new area of research has been developed to meet these demands, the Philosophical and Theoretical Psychology. With the aim of providing an introduction to this area, we highlighted some of the main journals, graduate programs and institutions that are involved in the production of knowledge in this field. We tried to establish the scope and purposes of the Theoretical and Philosophical Psychology. We reviewed different authors who sought to establish the main activities in this field. Thus, we provide some examples of important research, and we concluded that the encouragement of this kind of inquiry is indeed relevant to Psychology.
\end{abstract}

Keywords: theoretical psychology; philosophical psychology; conceptual analysis.

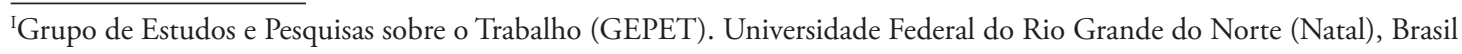

A falta de pesquisa teórica e de análises conceituais em Psicologia tem sido apontada reiteradamente como um problema por diferentes autores (Machado, Lourenço, \& Silva, 2000). Wittgenstein (1996), em uma passagem célebre, apontou que "existem na Psicologia métodos experimentais e confusão conceitual” (p. 206). Harré e Secord (1972) reclamaram da ingenuidade conceitual dos psicólogos e da sua tendência a substituir discussóes conceituais por definiçóes operacionais e experimentos, lamentando que a precisão de significado dos conceitos não seja tão valorizada no campo.

Moscovici (1972) afligiu-se pelo estado de negligência em que a pesquisa teórica se encontra em Psicologia, particularmente agora que "o sonho positivista de uma ciência sem metafísica” (p. 22) parece cada vez mais um sonho e não uma possibilidade real. Para Moscovici (1972), o resguardo do debate teórico em Psicologia possui aspectos emocionais, pois há um medo real de cair em debates filosóficos. No entanto, se pesquisadores de outras áreas podem se dividir em teóricos e experimentalistas, deixando mais liberdade para que cada atividade desenvolva a sua própria cultura, então porque náo pode haver a realizaçáo de algo assim também na Psicologia? ${ }^{1}$

Wachtel (1980) reclamou da falta de incentivos para a realização de trabalhos teóricos em Psicologia, apesar da sua evidente necessidade, e Wakefield (2007) recentemente retomou tal artigo para afirmar mais uma vez a necessidade de atividade teórica em Psicologia. Toulmin e Leary (1985) relacionam o estreitamento dos pensamentos teórico e filosófico em Psicologia com a influência positivista, que teve como resultado a formação de um verdadeiro culto ao empirismo na Psicologia.

1 O exemplo que costuma ser lembrado prontamente é o da Física Teórica (Howard, 1998). Ver a crítica de Robinson (2007), o que argumenta que o trabalho teórico na Física é completamente diferente daquele na Psicologia e esse tipo de comparação não deve ser feita. De toda maneira, Robinson (2007) concorda que uma "metapsicologia" dedicada a questōes conceituais é necessária. 
Alston (1985) destacou que a análise conceitual é uma parte importante de qualquer empreendimento intelectual, e a relaçáo entre filosofia analítica e teoria psicológica pode render frutos para a área em análise. Danziger (1985) indicou a excessiva valorização de questóes sobre métodos em detrimento de discussão teórica, ressaltando que alguns dos autores mais influentes da história da Psicologia, como Wundt, Freud, Köhler, Wertheimer, Lewin e Piaget, não fizeram uso de estatística inferencial para chegar a suas conclusóes.

Machado et al. (2000) discorreram de forma longa e convincente sobre como a Psicologia em geral tem se orientado predominantemente para fatos e pesquisas empíricas, dando pouca atenção à atividade fundamental de análise dos conceitos. Ao final, recomendam que o estudo da história da ciência, da lógica e da filosofia analítica pode ser mais benéfico do que as atuais disciplinas de estatística e dos métodos de pesquisa.

Machado e Silva (2007) mostraram como a análise conceitual é uma parte do método científico na Física, por meio de um estudo das análises conceituais feitas por Galileu, e recomendaram que a Psicologia invista nessa estratégia a fim de clarificar significados, expor problemas em modelos teóricos, revelar pressupostos náo explicitados e avaliar a consistência de teorias. Banicki (2011) postulou que a atividade de análise conceitual em Psicologia não tem sido desenvolvida como poderia, argumentou contra uma série de objeçóes feitas à análise conceitual, e concluiu que esta é uma ferramenta promissora que pode contribuir para o desenvolvimento da área.

Laurenti (2012) atualizou o diagnóstico de Wittgenstein, afirmando: "na Psicologia, existem métodos (experimentais e não experimentais) e confusão conceitual" (p. 179). Isto é, mesmo com as críticas ao método experimental e o surgimento de novos métodos, a desvalorização do trabalho teórico frente ao empírico continua forte.

Para Laurenti (2012), são diversos os problemas para aqueles que desejam conduzir pesquisas conceituais em Psicologia. Poucos orientadores aceitam trabalhos deste tipo, há dificuldades de apoio financeiro, de estabelecimento das linhas de pesquisa e de inserção em linhas existentes, além daquela de publicação em periódicos, o que faz com que alguns estudantes migrem para a pós-graduação em Filosofia.

Cruz (2013) defende a importância e a atualidade de se defender uma atitude filosófica na Psicologia, em um momento em que a medicalizaçáo e o sucesso das neurociências pressionam no sentido de reduzir o psicológico ao biológico. Para isso, aponta, como instrumentos especialmente importantes, a história dos conceitos de Canguilhem e o trabalho de Foucault, a fim de pensar a dimensão histórica do saber psicológico.

Os constantes e contínuos apontamentos de que a Psicologia precisa de mais trabalhos teóricos e conceituais são sintomáticos, tanto da sua escassez quanto de seu caráter marginal/minoritário. Os trabalhos acadêmicos teóricos em Psicologia existentes são frequentemente ignorados e desconhecidos.

Dado o contexto esboçado, o presente artigo pretendeu ser uma introdução ao campo, a fim de possibilitar uma primeira aproximação ao assunto, fornecendo indicaçôes sobre a sua formação e o seu progresso político-institucional e sua relevância dentro da Psicologia enquanto área do conhecimento como um todo.

\section{A formação da Psicologia Teórica e Filosófica no plano internacional e no Brasil}

O campo da Psicologia Teórica e Filosófica é reconhecido internacionalmente como uma área de pesquisa. Desde 1963, a American Psychological Association (APA) tem uma divisão voltada para a área, denominada Society for Theoretical and Philosophical Psychology. No início da década de 1980, fundou-se, também com projeção mundial, a International Society for Theoretical Psychology. Alguns periódicos são dedicados exclusivamente ao campo, como o "Journal of Theoretical and Philosophical Psychology", cujo primeiro número foi lançado em 1981²; o "New Ideas in Psychology", que começou a ser editado em 1983 e o "Theory and Psychology", cujo início se deu em 1991.

O estágio avançado da discussão sobre uma Psicologia Teórica e Filosófica nos Estados Unidos pode ser averiguado pelo debate sobre o artigo de Slife e Williams (1997). Os autores sugeriram a criação de uma subdisciplina de Psicologia Teórica como um reconhecimento de uma área que, na prática, já existe, e citam a fragmentação da Psicologia, as influências de perspectivas pós-modernas e as mudanças na filosofia da ciência como fatores que tornam a reflexão teórica extremamente necessária. $\mathrm{O}$ texto gerou diversas respostas, as quais serão resumidas no presente trabalho ${ }^{3}$.

2 O contexto de surgimento do periódico começou em 1966 quando uma newsletter começou a ser realizada pela Divisão 24 da APA (Teo, 2009).

3 Todas foram publicadas no mesmo número do periódico American Psychologist. Apenas o texto de Meux (1998), o qual parece refletir uma preocupação mais específica com axiologia, e a resposta de Slife e Williams (1998) às críticas, que não acrescenta muito ao artigo original, não foram comentados. 
Quinn (1998) defende que a Psicologia Teórica pode ser útil para a Psicoterapia, já que esta tem tradicionalmente criado várias escolas de pensamento e poderia se beneficiar de mais avaliação crítica de métodos e ideias. Abi-Hashem (1998) nota na Psicologia Teórica uma oportunidade para contrabalancear o caráter demasiado específico das pesquisas acadêmicas em Psicologia. No entanto, o investigador alerta para a possibilidade de que uma Psicologia Teórica pode ser tanto um modo de se obter uma visão mais global dos problemas como agravar a fragmentaçáo, já que aumenta o número de subdisciplinas (questão também levantada por Gantt, 1998).

Grace e Ferreras (1998) consideram que o argumento de Slife e Williams (1997) não tem sustentação, dado que a Psicologia vem funcionando bem sem uma subdisciplina de Psicologia Teórica e que a atividade teórica já está embutida na atividade normal de qualquer psicólogo. Weems (1998) demonstra preocupação de que uma Psicologia Teórica vista como subdisciplina acabe aumentando a distância entre a reflexão teórica e a pesquisa experimental. Este autor pondera no sentido de que seria melhor abordar a Psicologia Teórica como uma metadisciplina, a qual atravessa e engloba todas as disciplinas, sem necessariamente constituir uma área nova.

Stam (1998) reconhece que as diferentes atividades propostas por Slife e Williams (1997) são relevantes, porém argumenta que o papel da teoria em Psicologia não pode ser somente o de servir às pesquisas existentes, mas deve ser associado ao conhecimento histórico e à possibilidade de mudar a disciplina.

Howard (1998) afirmou que os "psicólogos estáo se afogando em um mar de dados que poucos tentam juntar" (p. 69) e que a síntese de achados é tão importante quanto o desenvolvimento de novos dados. Para este autor, a criação de uma nova subdisciplina não é a mais relevante, deve-se estimular trabalhos que tentem integrar conjuntos de experimentos.

É uma tarefa difícil, mas bastante recompensadora - afinal, o procedimento de coletar dados náo deve ser visto como um fim em si mesmo, mas como um para se adquirir conhecimento, que só pode advir pelo estabelecimento de vínculos com a teoria, que poderá fornecer aos dados empíricos um quadro de referência que lhes forneça coerência e estrutura (Howard, 1998).

Brand (1998) alertou para o que considera ser um viés pós-moderno do artigo de Slife e Williams (1997), ou seja, a defesa de que todo empreendimento científico se baseia em uma linguagem cujo sentido depende do contexto e das relaçóes sociais. Brand (1998) defende que uma Psicologia Teórica é importante, desde que não atue como veículo para um determinado modo de pensar (pós-moderno), e deve incluir também a atividade teórica positivista, isto é, que considera fundamental o papel de observaçóes empíricas. Mais importante do que estabelecer se a caracterização de Brand do pensamento pós-moderno é exata ou se o seu apelo ao empirismo é consistente, o ponto fundamental é que grupos com diferentes filosofias da ciência têm interesse em trabalho teórico, e não devem ser excluídos a priori.

Em face das análises apresentadas, e em diálogo com elas, considerou-se que os diferentes níveis de atividade teórica levantados ao longo desta discussão náo são excludentes. Em um primeiro nível, esta tarefa é inerente ao trabalho de todo e qualquer psicólogo; em um segundo, é uma atividade fim na qual alguns se engajam com mais afinco, seja em continuidade ou em paralelo a suas pesquisas empíricas; e em um terceiro nível, é uma que adquire autonomia, ocupando um lugar que não se define mais por sua relação com um trabalho empírico (seja de introdução, fundamentação, complementação, digressão etc.), mas que se afirma e entende como uma pesquisa de pleno direito, com sua própria ordem de questóes e problemas.

Portanto, a proposta de uma Psicologia Teórica e Filosófica não significa substituir as atividades tipos 1 e 2 descritas ou destacá-las de seu lugar de direito para concentrá-las em um outro lugar. A proposta é somar a essas duas um tipo de atividade 3, e a justificação para isso é que ela é caracterizada por um nível de autonomia que permite criar uma cultura de trabalhos teóricos capazes de entregar resultados que dificilmente seriam atingidos, ou levariam muito tempo para serem atingidos, se perseguidos somente por atividades de tipos 1 e 2 .

Independentemente da criação de uma subdisciplina, que possivelmente é uma questão relacionada ao número de psicólogos com interesse em promover tal ação no plano histórico e político, entende-se como um resultado conclusivo desse debate o fato de a maioria dos autores concordar com a relevância do desenvolvimento de pesquisas que procurem trabalhar questôes conceituais, filosóficas e teóricas dentro do campo da Psicologia.

No Brasil, embora pesquisas teóricas tenham sido realizadas, a grande maioria não parece ter consciência explícita de estar trabalhando em um campo distinto que merece reconhecimento e autonomia. 
Um forte sinal dessa falta de elaboração do campo é o fato de que não há periódicos especializados nesse domínio de produção acadêmica.

Uma mudança importante no cenário brasileiro foi a fundação, em 2010, do Núcleo de Pesquisa em História e Filosofia da Psicologia (NPHFP) da Universidade Federal de Juiz de Fora (UFJF). O mesmo grupo inaugurou em 2011 uma linha de pesquisa voltada para a História e Filosofia da Psicologia, no programa de pós-graduação em Psicologia da UFJF, que foi apresentada como a primeira do Brasil em seu gênero (Araujo, 2012; Araujo, Caropreso, Simanke, \& Castañon, 2013).

A atividade teórica/filosófica em Psicologia tem tradicionalmente estado associada à área de história, tanto no âmbito da pesquisa (programas de pós-graduação que envolvem os dois temas) quanto do ensino, por meio da disciplina de Teorias e Sistemas em Psicologia (Brock, 1998). O programa da UFJF segue o exemplo de outros, tais como: History and Theory of Psychology, da Universidade de York (Canadá); History and Theory of Psychology, da Universidade de Edimburgo (Escócia); Theory and History of Psychology, da Universidade de Groningen (Holanda). Um outro importante que não segue o padrão de associação com História em seu título é o da Universidade de Calgary (Canadá), denominado Social and Theoretical Psychology.

Institucionalmente, também há associação entre atividade teórica/filosófica e História em algumas sociedades de Psicologia. Dentro da Canadian Psychological Association, existe a seção History and Philosophy, que edita o periódico "History and Philosophy of Psychology Bulletin”. Na British Psychological Society, também existe uma seção intitulada History and Philosophy, que edita o periódico "History and Philosophy of Psychology". Já a APA possui a divisão 24, que é dedicada somente a Theoretical and Philosophical Psychology, separada da 26, chamada Society for the History of Psychology.

No Brasil, os grupos de trabalho (GT) da Associação Nacional de Pesquisa e Pós-Graduação em Psicologia (ANPEPP) se aproximam mais das seçôes das sociedades citadas, embora estes náo tenham a mesma estabilidade no que diz respeito à numeração e existência, ocorrendo variaçóes entre os diferentes encontros. No que diz respeito a esses GT, destaca-se a existência da atuação de um GT em História da Psicologia desde 1996. No entanto, não há um voltado especificamente à atividade teórica-filosófica.
Até esta parte do trabalho, foi apontado para a importância desta área de Psicologia e sua constituição no plano internacional e para o seu desenvolvimento incipiente no Brasil. No entanto, é preciso explorar com mais detalhes o que entendemos que uma Psicologia Teórica e Filosófica pode, ou deveria fazer, e o que ela não é ou quais atividades não estão dentro deste domínio.

\section{A atividade da psicologia teórica e filosófica}

Uma confusão que a proposta deste campo pode causar é a de que se trata de uma nova escola ou um paradigma, a partir do qual se deveria ler toda a Psicologia, estando no nível de uma evolucionista ou sócio-histórica, por exemplo. A Psicologia Teórica e Filosófica se propóe como um campo aberto de reflexão e trabalho metateórico e filosófico dentro da Psicologia de uma forma geral, sem compromisso específico com uma agenda que não seja a de criar e cultivar uma cultura de pesquisa que permita dedicação exclusiva a esse tipo de trabalho, como uma opção possível e desejável na pesquisa em Psicologia.

Indivíduos dentro do campo têm posicionamentos dos mais variados, inclusive sobre quais atividades a Psicologia Teórica e Filosófica deveria realizar. Certamente, não é nenhuma surpresa que a concepção do campo se torne alvo de disputas e que diferentes visóes sejam apresentadas, dado que ele deve refletir necessariamente a fragmentação da própria Psicologia. Algumas dessas visóes sobre o que o campo é, presentes nos trabalhos de Slife (2000), Martin (2004), Teo (2009) e Castañon (2012), serão apresentadas.

Slife (2000) considera que o psicólogo teórico tem dois propósitos principais: formular e ajudar outros a articular teorias que, em última instância, serão testadas empiricamente, seja de maneira quantitativa ou qualitativa; examinar e ajudar outros psicólogos a examinar temas não empíricos que sejam relevantes para o seu trabalho.

Para Slife (2000), o psicólogo teórico pode atuar como um consultor que ajuda outros a examinarem e construírem teoricamente seus trabalhos, de uma maneira análoga talvez ao trabalho da prestação de serviços de um estatístico para um pesquisador que não domina determinados recursos. Isso ajudaria a mudar a imagem do psicólogo teórico, que muitas vezes é tido como um crítico mordaz, o qual não oferece soluçôes, mas apenas aponta problemas. A posição 
de crítico seria por demais confortável — é preciso ir além e interagir, proporcionando opçóes construtivas.

A interação proposta por Slife se daria não só entre pesquisadores profissionais, mas também entre psicólogos teóricos e comunidade como um todo. A partir da noção aristotélica de phronesis, o investigador defende que todo conhecimento envolve necessariamente um aspecto ético, de diálogo e de interação com a sociedade, e por isso, o psicólogo teórico deveria não só participar de publicações especializadas, como também escrever para o público leigo, recuperando a figura do intelectual público.

Martin (2004) divide as atividades teóricas da Psicologia Teórica em três grandes tipos: relacionadas diretamente à pesquisa empírica; associadas à prática da profissão (incluindo aspectos morais e políticos); relacionadas à presença da Psicologia na sociedade como um todo. Para Martin (2004), a Psicologia Teórica teria um papel terapêutico ao examinar a pesquisa, a prática profissional e as iniciativas públicas dos psicólogos, debatendo sua retórica, seus conceitos e fazendo dialogar diferentes pontos de vista e argumentos.

Teo (2009) defende que as psicologias teórico-filosóficas se caracterizam como programas metateóricos que trabalham em domínios filosóficos, assumindo perspectivas reflexivas sobre temas psicológicos. Para Teo (2009), todo psicólogo é teórico, no sentido de que faz uso necessariamente de teorias e contribui para elas. O que caracteriza o diferencial da atividade metateórica é a reflexão sobre os pressupostos implícitos e explícitos das teorias dentro do âmbito filosófico.

Teo (2009) considera que as áreas filosóficas relevantes seriam: Ontologia, que engloba discussóes como o objeto da Psicologia, a relaçáo mente e corpo e a natureza das categorias psicológicas; Epistemologia, que aborda natureza do conhecimento e verdade nas teorias psicológicas; ética/prática, para discussóes sobre fato e valor, justiça social etc.; e estética, que seria pertinente pela utilização da arte na investigação da subjetividade.

O mesmo investigador cita ainda que trabalhos metateóricos podem ser de três tipos: reconstrução, que realizaria a integração e a avaliação de teorias por meios históricos, teóricos ou conceituais; desconstrução, ou seja, a crítica de uma determinada teoria, ou construção, que é a apresentação de uma teoria nova e original.

Castañon (2012) entende que a Psicologia Filosófica pode ser definida, de um modo geral, como o estudo das questóes conceituais em Psicologia, di- vidindo-a em quatro campos: Epistemologia da Psicologia, que engloba os problemas fundamentais da Ontologia e da Epistemologia da Psicologia; Psicologia Teórica, que assume como pressuposto a aplicaçáo da ciência moderna ao problema psicológico e funciona como um correlato da física teórica; Psicologia Filosófica, um campo difuso que seria caracterizado pela abordagem de temas psicológicos sem o uso do método científico; Filosofia da Mente, dedicada aos fenômenos mentais, como consciência, memória, senso/percepção, conceitualização, dentre outros, e sua relação com o cérebro.

Essas diferentes formas de dividir o campo revelam a riqueza de suas possibilidades. Propóe-se aqui que a diferença fundamental seja entre, de um lado, a psicologia teórica concebida como auxiliar da pesquisa empírica - correspondente ao sentido 1 de Slife, 1 de Martin e 2 de Castañon - e a psicologia teórica concebida de maneira mais ampla, englobando uma série de atividades e problemas de difícil categorizaçáo - sentido 2 de Slife, 2 e 3 de Martin, 1, 3 e 4 de Castañon e talvez toda a proposta metateórica de Teo.

Embora ambas as formas tenham legitimidade e muito trabalho a fazer, gostaríamos de discorrer mais longamente sobre a Psicologia Filosófica em seu sentido mais amplo, que não se considera diretamente ligada a um modo específico de pesquisa empírica ou ao empreendimento de aplicação da concepção moderna de ciência à Psicologia. Tornar-se o auxiliar de um determinado modo de "pesquisa empírica" nos parece potencialmente problemático, dado que pode significar assumir alguns pressupostos sobre o que é "empírico", sem o devido exame crítico. Este é considerado especialmente necessário e útil diante do contexto atual, de tantas transformaçóes na filosofia da ciência.

$\mathrm{O}$ que antes era concebido apenas como filosofia da ciência, atualmente se tornou um campo de estudos muito mais sofisticado, no qual história, filosofia e sociologia da ciência vieram a trabalhar de forma mais integrada (Fagerberg, Landström, \& Martin, 2012; Cetina, 1991). O desenvolvimento de pesquisas sobre fazer ciência (Martin, Nightingale, \& Yegros-Yegros, 2012; Köche, 2005) vem demonstrando consistentemente há anos uma pluralidade enorme de métodos, culturas, linguagens e metafísicas no fazer científico.

No entanto, muitos psicólogos continuam se guiando por uma versão limitada, simplificada e cristalizada de um método científico fundado exclusivamente na elaboração de hipóteses testáveis, na 
operacionalização para a verificação de tais hipóteses, na utilização de ferramentas estatístico-inferenciais e/ ou de resumo de informaçáo e discussáo de resultados à luz dos novos dados produzidos. A aplicação mecânica de um procedimento metodológico e a obsessão da Psicologia com o método foram retratadas como metodolatria (Danziger, 2002) e fetichismo metodológico (Koch, 1981).

O currículo de graduação nem sempre tem conseguido acompanhar as mudanças, limitando-se muitas vezes a considerar que as grandes perspectivas teóricas para abordar a Psicologia já foram realizadas e não há mais a necessidade de procurar (Slife $\&$ Williams, 1997). As últimas décadas assistiram ao surgimento de grandes abordagens teóricas inovadoras em Psicologia, como o construcionismo social de Gergen (1973; 1985; 1996; 2009), a psicologia narrativa (Brockmeier \& Harré, 2003; Murray, 2003) e todo um conjunto de abordagens discursivas (Potter, 2003; Edwards, 2004) e pós-modernas (Kvale, 1992).

Porém, não são somente as grandes reformulações epistemológicas da Psicologia, o avanço geral dos estudos sobre a ciência e as perspectivas pós-modernas que exigem trabalho teórico por parte dos psicólogos. Estudos teóricos específicos têm o potencial de provocar debates e mudanças significativas no modo de se praticar ciência em Psicologia. Exemplos disso são os debates a respeito da apropriação do conceito de "definiçáo operacional" pela Psicologia (Koch, 1999; Feest, 2012), a utilização do teste da hipótese nula (Bakan, 1966; Meehl, 1978; Cohen, 1994; Gigerenzer, 2004; Lambdin, 2012) e o conceito de mensuração utilizado usualmente em Psicometria (Michell, 1997; 2000; 2003; 2006; Sturm, 2012).

\section{Consideraçóes finais}

A Psicologia não é uma ciência tão independente da Filosofia ou mesmo ambicionaria, em seu esforço histórico, para se constituir enquanto ciência "respeitável" no contexto das demais ciências (Koch, 1981). No entanto, a especificidade de seus temas e os problemas epistemológicos, teóricos e metodológicos que estes geram exigem um conhecimento da área que filósofos não costumam ter, além do saber de filosofia e abertura para reflexão que muitos psicólogos acostumados a pesquisas empíricas tampouco possuem. Por isso, compartilhamos com muitos dos autores citados ao longo deste artigo a ideia de que a Psicologia necessita de uma área teórica e filosófi- ca para tratar convenientemente os problemas que lhes são próprios.

O movimento teórico em Psicologia tem passado relativamente despercebido pela maior parte dos psicólogos, em função de seu caráter excessivamente centrado no método das pesquisas em Psicologia. A ideia de que o método é o componente essencial e garantidor da "verdade" ou "validade" continua com grande força dentro da Psicologia acadêmica (Slife \& Williams, 1997). Se a pesquisa teórica não for estimulada como uma forma possível de realizar trabalhos acadêmicos em Psicologia, a formulação dos problemas e a busca por respostas correm o risco de continuar sendo atividades guiadas principalmente por questôes metodológicas desconectadas de reflexão, que ultrapassem o domínio interno de validade dos procedimentos utilizados - como é o caso das discussões técnicas envolvendo representatividade, viés de observação, controle e generalização.

A Psicologia guiada, especialmente, por preocupaçôes metodológicas nesse nível mais operacional está condenada à prática chamada por Kuhn (2009) de "ciência normal", desprovida de força e profundidade necessárias à formulação de questóes sobre o significado das suas escolhas, perguntas e, sobretudo, premissas de pesquisa. É isso o que a Psicologia Teórica e Filosófica tem para oferecer de mais precioso: a oportunidade de que psicólogos possam se dedicar em tempo integral a analisarem aspectos fundamentais e ordenadores da busca científica em nível de pressupostos centrais - talvez assimiláveis ao que Lakatos (1989) denominou de "núcleo duro" das propostas teóricas. Esperamos que o presente artigo possa ter chamado atenção para este tema e ajudado a fundamentar um maior interesse nessa direção.

\section{Referências}

Abi-Hashem, N. (1998). Returning to the fountains. American Psychologist, 53(1), 63-64.

Alston, W. P. (1985). Conceptual analysis and psychological theory. In: S. Koch \& D. E. Leary, A century of psychology as science. (pp. 638-652). Washington: APA.

Araujo, S. F. (Org.). (2012). História e filosofia da Psicologia: perspectivas contemporâneas. Juiz de Fora: UFJF.

Araujo, S. F., Caropreso, F. S., Simanke, R. T., \& Castañon, G. A. (2013). The Wilhelm Wundt Center and the first graduate program for the history and philosophy of psychology in Brazil: a brief report. History of Psychology, 16(3), 222-224. 
Bakan, D. (1966). The test of significance in psychological research. Psychological Bulletin, 66(6), 423-437.

Banicki, K. (2011). Connective conceptual analysis and psychology. Theory \& Psychology, 22(3), 310-323.

Brand, J. L. (1998). Theoretical psychology, Yes; methodological egalitarianism, No! American Psychologist, 53(1), 66-67.

Brock, A. (1998). Pedagogy and research. The psychologist, 11, 169-171.

Brockmeier, J. \& Harré, R. (2003). Narrativa: problemas e promessas de um paradigma alternativo. Psicologia reflexão e crítica, 16(3). Recuperado de: http://www.scielo.br/pdf/prc/ v16n3/v16n3a11.pdf

Castañon, G. A. (2012). Filosofia da psicologia: uma taxonomia. In: S. F. Araujo (2012), História e filosofia da psicologia: perspectivas contemporâneas. (pp. 187-221). Juiz de Fora: Universidade Federal de Juiz de Fora.

Cetina, K. K. (1991b). Merton's sociology of science: the first and the last sociology of science? Contemporary Sociology, 20(4), 522-526.

Cohen, J. (1994). The earth is round $(\mathrm{p}<.05)$. American Psychologist, 49(12), 997-1003.

Cruz, M. G. A. (2013). A defesa de uma atitude filosófica na psicologia: breve reflexão sobre a prática psi e o processo de medicalização. Filogenese, 6(1), 78-92.

Danziger, K. (1985). The methodological imperative in psychology. Philosophy of the Social Sciences, 15, 1-13.

Danziger, K. (2002). Constructing the subject: historical origins of psychological research. Cambridge: Cambridge University Press.

Edwards, D. (2004). Psicologia discursiva: unindo teoria e método com um exemplo. In: L. Íñiguez (Org.) (2004), Manual de análise do discurso em ciências sociais. (pp. 181-205). Petrópolis: Vozes.

Fagerberg, J., Landström, H., \& Martin, B. R. (2012). Exploring the emerging knowledge base of 'the knowledge society'. Research Policy, 41, 1121-1131.

Feest, U. (2012). O operacionalismo na psicologia: sobre o que é o debate, sobre o que deveria ser o debate. In: S. F. Araujo (2012). História e filosofia da psicologia: perspectivas contemporâneas. (pp. 259-296). Juiz de Fora: Universidade Federal de Juiz de Fora.

Gantt, E. E. (1998). Some questions about theoretical psychology. American Psychologist, 53(1), 65-66.

Gergen, K. J. (1973). Social Psychology as History. Journal of Personality and Social Psychology, 26(2),
309-320. Recuperado de http://www.swarthmore. edu/Documents/faculty/gergen/soc_psych.pdf

Gergen, K. J. (1985). The Social Constructionist Movement in Modern Psychology. American Psychologist, 40(3). Recuperado de: http://www. swarthmore.edu/Documents/faculty/gergen/Social_ Constructionist_Movement.pdf

Gergen, K. J. (1996). Social Psychology as Social Construction: The Emerging Vision. Recuperado de: http://www.swarthmore.edu/Documents/ faculty/gergen/Social_Psychology_as_Social_ Construction_The\%20Emerging_Vision.pdf

Gergen, K. J. (2009). An invitation to social construction. London: Sage.

Gigerenzer, G. (2004). Mindless statistics. The Journal of Socio-Economics, 33, 587-606.

Grace, R. C. \& Farreras, I. G. (1998). Are theoretical consultants needed? American Psychologist, 53(1), 67-68.

Harré, R. \& Secord, P. F. (1972). The explanation of social behavior. Totowa: Rowman and Littlefield.

Howard, R. W. (1998). Every psychologist needs to be a theorist. American Psychologist, 53(1), 69-70.

Koch, S. (1981). The Nature and limits of psychological knowledge: Lessons of a century qua "science". American Psychologist, 36(3), 257-269.

Koch, S. (1999). Psychology's Bridgman versus Bridgman's Bridgman: a study in cognitive pathology. In: S. Koch (1999), Psychology in human context. Essays in dissidence and reconstruction. (pp. 366393). Chicago: The University of Chicago Press.

Köche, J. C. (2005). Pesquisa cientifica. Critérios epistemológicos. Petrópolis: Vozes.

Kuhn, T. S. (2009). A estrutura das revoluçóes cientificas (Trad. Beatriz Vianna Boeira e Nelson Boeira). São Paulo: Perspectiva. (Texto originalmente publicado em 1962).

Kvale, S. (Ed.) (1992). Psychology and postmodernism. London: Sage.

Lakatos, I. (1989). The methodology of scientific research programmes - philosophical papers, vol.1. Cambridge: Cambridge University Press.

Lambdin, C. (2012). Significance tests as sorcery: science is empirical - significance tests are not. Theory \& Psychology, 22(1), 67-90.

Laurenti, C. (2012). Trabalho conceitual em psicologia: pesquisa ou "perfumaria"? Psicologia em Estudo (Maringá), 17(2), 179-181.

Machado, A., Lourenço, O., \& Silva, F. J. (2000). Facts, concepts, theories: the shape of psychology's epistemic triangle. Behavior and Philosophy, 28, 1-40. 
Machado, A. \& Silva, F. J. (2007). Toward a richer view of the scientific method: the role of conceptual analysis. American Psychologist, 62(7), 671-681.

Martin, J. (2004). What can theoretical psychology do? Journal of Theoretical and Philosophical Psychology, 24(1), 1-13.

Martin, B. R., Nightingale, P., \& Yegros-Yegros, A. (2012). Science and technology studies: exploring the knowledge base. Research Policy, 41, 1182-1204.

Meehl, P. (1978). Theoretical risks and tabular asterisks: sir Karl, sir Ronald, and the slow progress of soft psychology. Journal of Consulting and Clinical Psychology, 46, 806-834.

Meux, M. O. (1998). A value agenda for theoretical psychology. American Psychologist, 53(1), 64-65.

Michell, J. (1997). Quantitative science and the definition of measurement in psychology. British Journal of Psychology, 88, 355-383.

Michell, J. (2000). Normal science, pathological science and psychometrics. Theory \& Psychology, 10(5), 639-667.

Michell, J. (2003). The quantitative imperative: positivism, naïve realism and the place of qualitative methods. Theory \& Psychology, 13(1), 5-31.

Michell, J. (2006). Psychophysics, intensive magnitudes, and the psychometricians' fallacy. Studies in History and Philosophy of Biological and Biomedical Sciences, 17, 414-432.

Moscovici, S. (1972). Society and theory in social psychology. In: J. Israel \& H. Tajfel (Eds.) (1972), The context of social psychology: a critical assessment. (pp. 17-68). London: Academic Press.

Murray, M. (2003). Narrative psychology and narrative analysis. In: P. M. Camic, J. E. Rhodes, \& L. Yardley (Ed.) (2003). Qualitative research in psychology. Expanding perspectives in methodology and design. (pp. 95-112). Washington: APA.

Potter, J. (2003). Discourse analysis and Discursive Psychology. In: P. M. Camic, J. E. Rhodes, \& L. Yardley (Ed.) (2003), Qualitative research in psychology. Expanding perspectives in methodology and design. (pp. 73-94). Washington: APA.

Quinn, M. H. (1998). The role of theory in psychotherapy. American Psychologist, 53(1), 71.

Robinson, D. N. (2007). Theoretical Psychology. What is it and who needs it? Theory and Psychology, 17(2), 187-198.

Slife, B. D. (2000). The practice of theoretical psychology. Journal of Theoretical and Philosophical Psychology, 20(2), 97-115.
Slife, B. D. \& Williams, R. N. (1997). Toward a theoretical psychology: Should a subdiscipline be formally recognized? American Psychologist, 52(2), 117-129.

Slife, B. D. \& Williams, R. N. (1998). Theoretical psychology as a subdiscipline: the conversation continues. American Psychologist, 53(1), 71-72.

Stam, H. J. (1998). Theory and disciplinarity. American Psychologist, 53(1), 70.

Sturm, T. (2012). Há algum problema com a psicologia matemática no século dezoito? Um novo olhar sobre o velho argumento de Kant. In: S. F. Araujo (2012). História e filosofia da psicologia: perspectivas contemporâneas. (pp. 87-132). Juiz de Fora: Universidade Federal de Juiz de Fora.

Teo, T. (2009). Editorial. Journal of Theoretical and Philosophical Psychology, 29(1), 1-4.

Toulmin, S. \& Leary, D. E. (1985). The cult of empiricism in psychology, and beyond. In: S. Koch \& D. E. Leary (1985). A century of psychology as science. (pp. 594-617). Washington: APA.

Wachtel, P. L. (1980). Investigation and its discontents: some constraints on progress in psychological research. American Psychologist, 35(5), 399-408.

Wakefield, J. C. (2007). Why psychology needs conceptual analysts: Wachtel's 'discontents' revisited. Applied and Preventive Psychology, 12, 39-43.

Weems, C. F. (1998). Theoretical psychology: subdiscipline or metadiscipline? American Psychologist, 53(1), 68-69.

Wittgenstein, L. (1996). Investigaçôes filosóficas. (Trad. José Carlos Bruni). São Paulo: Nova Cultural.

\section{Endereço para correspondência:}

Flávio Fernandes Fontes

Universidade Federal do Rio Grande do Norte

Grupo de Estudos e Pesquisas sobre o Trabalho

Campus Lagoa Nova

CEP: 59072-970 - Natal/RN

E-mail: flaviofontes@outlook.com

Recebido em: 19/12/2014

Revisto em: 04/03/2015

Aceito em: 27/05/2015 